\title{
MOBEEZE. Natural Interaction Technologies, Virtual Reality and Artificial Intelligence for Gait Disorders Analysis and Rehabilitation in Patients with Parkinson's Disease
}

\author{
${ }^{1}$ FIDESOL (Free Software R\&D Foundation) (Spain) \\ ${ }^{2}$ Virtualware Group (Spain)
}

Lombardo, J.M. ${ }^{1 *}$, Lopez, M.A. ${ }^{1}$, Lopez, M. ${ }^{1}$, León, M. ${ }^{1}$, Miron, F. ${ }^{1}$, Arranbarri, J. ${ }^{2}$, Alvarez, D. ${ }^{1}$

Received 23 April 2019 | Accepted 30 April 2019 | Published 9 July 2019

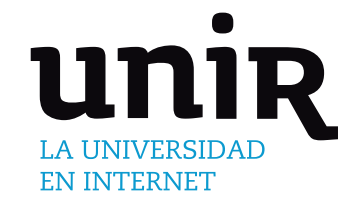

KEYWORDS

Parkinson, Freezing, Natural Interaction, Virtual Reality, Artificial Intelligence, Cognitive Computing.
Parkinson's Disease (PD) is the most common degenerative disorder after Alzheimer's disease. Generally affecting elderly groups, it has a strong limiting effect on physical functioning and performance of roles, vitality and general perception of health. Since the disease is progressive, the patient knows he's going to get worse. The deterioration is significant not only in mobility but also in pain, social isolation, and emotional reactions. Freezing is a phenomenon associated with this disease and it is characterized by a motor disorder that leaves the patient literally stuck to the ground. Mobeeze is designed with the main objective of providing health personnel with a tool to analyse, evaluate and monitor the progress of patients' disorders as well as the personalization and adaptation of rehabilitation sessions in patients with Parkinson's disease. Based on the characteristics measured in real time which will allow the strengthening effects of rehabilitation and help to assimilate them in the long term. The creation of Mobeeze allows the constitution of a system of analysis and evaluation of march disorders in real time, through natural interaction, virtual reality and artificial intelligence. In this project, we will analyse if these non-invasive technologies reduce the stress induced to the patient when he is feeling evaluated.

\section{INTRODUCTION}

A VAILABLE data concerning Parkinson Disease (PD) indicates -prevalence over other neurodegenerative diseases with relation to ageing and treatment complexity. The disease is estimated to increase in the next few years on a global level making it the second most prevalent neuro-digestive disease right after Alzheimer's disease.

Parkinson's Foundation in the United States, where more than 60 thousand cases are diagnosed every year, estimates that 1 million people are affected by Parkinson only in this country and more than 10 million are affected worldwide.

The European Parkinson's Disease Association (EPDA) gave a lower estimate, pointing out that there are 6.3 million persons affected by PD worldwide; nevertheless, the number is equally disturbing. In Spain alone, the numbers are estimated between 100 to 150 cases of persons suffering from Parkinson's Disease. According to the Spanish Brain Foundation like EPDA, diagnosing today 10 thousand new cases each year, the number that is intended to double in the next 20 years and triple in the year 2050. This confirms the evaluation realized by the Spanish Society of Neurology.

More than one third of the patients with PD (mostly the ones in advanced stages of the disease) suffer from a well-known phenomenon

* Corresponding author.

E-mail address: jmlombardo@fidesol.org known as "freezing". This is a temporary motor disorder that lasts seconds and is characterized by the sensation of "feet glued to the floor". It is mostly felt when the patient tries to start or resume his or her walk, turning to change direction or pace of the walk. It can also appear by just passing through a door or through narrow spaces like corridors, also specially appearing in stress episodes or with distractions. This may cause uncontrolled falls.

In the last few years giant leaps have been taken to better the standard of living of the patients of neurological diseases and related disorders such as PD, but science goes on. Concerning patient's rehabilitation, therapies are centred on minimizing and/or delaying the effects of these disorders. These therapies normally derive in optimal results though not all patients can have access to them.

\section{A. Parkinson Patient Treatment Actual Methods}

As of today, PD doesn't have any cure. However, its symptomatology can be controlled in an adequate manner [1]. Researchers are presently working on the improvement of the symptoms associated with the neural loss and neural death from several points of view:

- Pharmacological: There's a whole set of medications that favour the dopamine production or delay its deterioration. It's administrated according to the gravity of symptoms. Less powerful drugs are administrated in the first stages followed by a progressive dosage increase.

- Surgical: Procedures centred on the intervention of the damaged part of the brain are only recommended in a very low percentage of patients, not more than $5 \%$. Patients benefited by this are 
only the ones with a very advanced PD case or null response to pharmacological treatment.

- Subthalamotomy: Elimination of the affected brain area and installation of a local pacemaker. This technique is also performed in cases of very advance disease or null response to pharmacological treatment.

- Physical Rehabilitation: A key aspect of living with the disease is the maintenance of muscle tone and motor functions, so daily physical activity supplemented by exercises to help maintain mobility in affected members is essential.

As regards to physical rehabilitation, there is not always a possibility for patients to carry out this therapy, so scenarios based on information technology is suggested as a possible solution.

\section{B. ICT Applied to the Study and Treatment of PD by MOBEEZE}

In recent years, the Information and Communications Technology (ICT) have contributed to solutions aimed at improving the healthcare quality which allows one to address the pathology through specialised systems in order to ensure a more focused attention on the patient.

It is suggested that the use of ICT for the treatment of PD could help to rehabilitate motor abilities, although further research to establish its clinical effectiveness and safety is necessary. In order to develop Mobeeze, there were taken into consideration various complementary technologies to address patient evaluation: (1) Virtual reality system (VR), in this case, VR goggles for training in immersive environments, (2) Natural interaction devices for analysing all variables related to patients' motion and manual dexterity. A three-dimensional virtual world recreation environment compatible with such devices is used. Information generated through training with these devices provides data such as the step length, speed, angle of both knees and hips, etc. Thanks to techniques of artificial intelligence (AI), there is new knowledge of medical research regarding the suitability of the application of these technologies.

The key elements for applying the VR neurorehabilitation are repetition, feedback and patient's motivation. Repetition is important for motor learning and neuroplastic changes to occur. These changes maintain long-term lessons learned but must be linked to a sensory feedback in the outcome of each of the embodiments. On the other hand, in order to perform time and again the activities required for neurorehabilitation, the subject motivation is fundamental. This is achieved by focusing the different activities as a video game so that the treatment sessions are much more enjoyable and attractive [2]. The use of VR as a supplemental to therapy includes other important advantages such as the ability to control each session accurately and repeatedly along with the ability to adapt the interfaces to the users' motor limitations and the recreation of secure virtual environments for practicing skills that would otherwise imply potential risk in the real world.

Despite all these advantages, there are some doubts about applying VR to neurorehabilitation, especially in regard to the equivalence between the movements in real and virtual environments. That is: the resulting functional capacity. Several studies have reached to the conclusion of relative equivalence between environments, although small deviations were accounted for due to different spatial perception [3]-[5].

In recent years, studies have been published on various neurological diseases in which the VR is applied: cerebrovascular disease [6], multiple sclerosis [7], in patients with sequelae after traumatic brain injury [8] and PD, which is the object of the current project.

Additionally, natural interaction devices (NI) can further enhance the immersion experience, allowing the gap with reality to become even lower. Some studies suggest that neurorehabilitation VR / NI could induce cortical reorganization which plays a key role in the recovery of motor [9] capacity and proves the transfer of acquired skills to the real world [10], which makes the VR itself a tool of great potential in neurorehabilitation.

\section{MATERIALS}

\section{A. System Virtual Immersion by Oculus Rift}

Oculus Rift (OR) is a VR device developed and marketed by Oculus VR company, a subsidiary of Facebook Inc., which launched to the market on late March 2016.

It uses an OLED panel for each eye, and technical specifications for the projection image are perfectly designed to create a threedimensional world where an immersive user experience is complete. It relies on two hardware devices, controllers to interact with objects in a three-dimensional environment and satellites that pinpoint the physical position of the user in order to interact with objects in threedimensional environment.

\section{Virtual Immersion in the eHealth Sector.}

In recent years, interest in the sector has increased due to the inclusion of this technology in therapies and interventions [11]. OR is growing popular in health centres and even private homes, while simplifying the development of its applications. Thanks to this, it can be considered to replace traditional therapies with novel and immersive ones, even both ambulatory and domiciliary treatments.

However, an important challenge for VR treatments is the need for more evidence to show that the benefits obtained from such treatments translate into real benefits.

\section{B. Natural Interaction System by Leap Motion}

Leap Motion (LP) is a sensor that draws a virtual image of hands and joints from the wrists to the distal phalanges of the fingers and also represents the image of hands on a computer screen recording these movements. LP is used in the project for patient manual ability evolution accounting for the freezing phenomenon.

\section{Monitoring System Activity by Kinect}

Although no longer marketed in 2017, following its launch in 2010, the Kinect device was a breakthrough in the use and application of technologies for natural interaction in the field of medicine and study procedures, monitoring and rehabilitation was conducted with patients, as is the case of Parkinson's patients. The introduction of Kinect in this area gave these therapies a low cost device that, thanks to its processing capacity of actions and obtaining information about them, along with the inclusion of a VR system, made possible the Immersion binding a Natural Virtual-Interface, a combination that would improve the patient's immersive experience.

Thus, different studies have sought to demonstrate the optimization of results in tests performed in a controlled healthcare environment for which the experience was designed [12] using the Kinect device. The aim of Mobeeze was to determine the therapeutic effectiveness of VR in patients with PD using Kinect as an instrument for the monitoring of the freezing phenomenon.

Various research groups have also oriented part of their research to test the efficiency of Kinect and the application of VR technology to monitor and rehabilitate of PD patients. In the case of Brunel University in London, where a positive application of the Kinect device was accounted for, orienting in the same manner their research for monitoring purposes and helping people affected by this phenomenon of motion disturbance, getting satisfactory results and conclusions [13] [14]. Likewise, the Cochrane Movement Disorders Group of 
the University of Lisbon conducted an independent research on VR exercise interventions for rehabilitation in PD [15].

In the case of the Mobeeze project, in which one of the goals is to identify the characteristic signs of PD motor deterioration relative to the patient's stage, Kinect was used as an affordable, reliable and validated tool for motion analysis.

\section{Interactive Motor of Cognitive Inference}

Cognitive computing can be considered, although not all researchers agree [16], emerged as a specific branch of AI, focusing on providing the machine a component of "understanding" that emulates the workings of the human mind. It could be defined based on a set of biologically inspired technologies, as well as those that mimic (and overcome) intelligent animal functions and easing interaction between humans and machines. IBM Watson [17] is one of the earlier examples.

Cognitive computing difficult to discern from AI itself, but rather a new way of naming it. It is not easy to differentiate between a good intelligent software and AI. John McCarthy, founder of AI, around 1955 said, "Once a program does something that previously only humans did, we stop calling it artificial intelligence.” Speech recognition is a good example for this, as well as the process of written text conversion, automatic translation or the ability to dictate to a computer. Five years ago, it was an unsolvable problem. Laboratories around the world were investigating it for decades. It is present today in our cell phones and nobody is surprised nor calling it artificial intelligence despite being a colossal achievement.

We tend to think of AI as a gigantic being that performs multiple human functions. However, cognitive computing is quite different: it is small and it's everywhere. Its power lies in its ubiquity. Along with these functionalities: image and face recognition. But there is much more, for example personality traits detection through social networks or message tone analysis.

Simplification or humanization of machine interaction machines is challenging. It's difficult to accept that in order to deal with a machine human have to understand their primitive system commands. The aim is that the machines understand humans and not vice versa.

In recent years, AI is gaining ground in terms of diagnostic procedures, monitoring, analysis as well as evaluation of patients. Concerning PD, for the Michael J. Fox Foundation (named after and created by the famous actor, whose mission is to find a cure for the disease and develop improved therapies), AI strategies are priority in finding treatments and a cure for PD. Thus, in 2013, the foundation launched a challenge data in order to generate a brainstorming in the development of solutions for monitoring and treatment of PD [18]. As part of this challenge, the proposal winner planted an approach to machine learning to address the problem, demonstrating the viability and value of collecting and analysing cell phone data to monitor Parkinson. This was obtained through cell phones incorporation and low-cost sensors.

On the other hand, and through some funds granted by the same foundation, IBM developed a prototype consisting in a small sensor nail for obtaining data, which could allow specialists to track, monitor and diagnose the movement disorders in people with PD. The extracted data are studied by AI algorithms that are taught a series of patterns [19].

The Parkinson and Movement Disorders Unit at the Polytechnic University of Catalonia together with Telefónica I+D, developed a system for monitoring patients with Parkinson called Rempark, which uses AI models to remotely control the patient, monitoring in order to help and manage their own mobility. Among its components, it has sensors to identify, in real time, freezing of gait and a guidance system of travel that provides auditory cues. The information obtained from the patient through the sensors and devices with embedded enables the specialist to receive accurate and relevant information from the patient in order to make decisions or treatment information.

Research on the design of AI models that provide relevant data and information on patients with PD have not only been aimed at monitoring and/or rehabilitation patients, but also for early detection and improved diagnoses of neurodegenerative diseases, especially Parkinson's disease. In order to improve the accuracy of interpretations and evaluations made by specialists, Mobeeze is using inference engines data, designed to provide the researcher with a knowledge basis by training patients in virtual reality environments and natural interaction, providing a new perspective and new data study.

\section{E. Environments Recreation of Virtual Worlds}

For the recreation of scenarios contemplated in the project, it is necessary to have a platform for creating three-dimensional virtual worlds that can also be compatible with the use of virtual reality goggles and natural interaction elements. This article is not intended to make a study of the possibilities, especially free software, since it said that the analysis was performed on another project with similar technical characteristics, Practica [20], but oriented to create a platform for training specialty in immersive environments to improve productivity. Therefore, the selected tool was Unity 3D development environment applications and video games widely accepted by the community 2D/3D environments. This framework supports a lot of peripherals, in particular those used in Mobeeze (Oculus Rift, Leap Motion and Kinect). Although Unity 3D is not a free software license, it allows free access to the community and is enriched with a repository of extensions provided by users of this technology, where you can find all kinds of resources (free or paid) high quality. It also supports the export of software to multiple platforms such as Android, Windows, iOS and even consoles like PlayStation.

\section{Methodology}

Mobeeze arises to experiment and analyse the viability of different technologies applied to carry out tracking people with PD, so it allows medical personnel to have a system that tracks and determines the degree of progress of the phenomenon of freezing. It also aims to support the research by incorporating a trained cognitive engine, taking as reference the historical training conducted by Mobeeze. Using this tool, we intend to conduct a study and careful monitoring of the patient so that training can be adjusted depending on the progress of this disorder in the patient making the evolution of the disease as slow as possible and therefore offering the patient a better quality of life. To this end, it has developed a tool for recreating situations as close to reality as possible using not only visual technologies based on VR, but also acoustic tech, which is tested on the patient in certain "stress" situations. The reactions are collected and stored so that, through a series of sessions, one can analyse the evolution of the patient. The configuration of the situations in which you can find a patient has been determined to be opposite to them in the closest form to real situations, increasing or decreasing the level of stress to which the patient undergoes. Thanks to the recreation of virtual reality environments, exposure to situations of high stress level that may not be viable in a real environment is allowed, as is the case in situations where a car crosses in front of the patient.

The training of these tests based on virtual reality technologies and natural interaction generates valuable information to track both during different sessions to further analysis. Based on this, the own patient's history and the history of workouts that were previously performed in other patients, healthcare workers can access the inference by a 
cognitive motor supplied with such information, including the data of the patient. To this end, it is designed in a similar way to a virtual assistant who, through a query interface-based chat, provides valuable information to adjust researcher training and determine the evolution of the disease component. It should be borne in mind, while incorporating this component at the end of the project course, it has not been possible to validate the results of using this cognitive inference engine and query interface by medical researchers participating in the project. However, we are currently working on determining the effectiveness of this component.

Therefore, Mobeeze consists of several phases: (1) acquisition of patient data and determining the training; (2) creation of virtual reality system and natural interaction to measure the performance of patients in the environment created by the system, (3) cognitive inference engine together with (4) a query interface.

\section{A. Acquisition of Patient Data}

This project created on one hand, an observational study to analyse the population characteristics and motor signs and not dependent engines disease was crated and on the other hand, two subsequent experimental studies in order to evaluate the effectiveness of the rehabilitation program based virtual reality to optimize the way in study population study:

\section{Observational Study}

The evaluation protocol for the observational study consisted of three parts, dedicating five to ten minutes per patient:

a) Initial interview through which data and pertinent information on the characteristics of the participants who took part in the study were collected.

b) Evaluation of motor signs, which had the following appraisals: gait, manual dexterity, gait freezing, manual freezing evaluation, freezing in a favourable virtual immersion environment, freezing in a freezing provoking virtual immersion environment.

c) Evaluation of non-motor signs applying a battery of scales and questionnaires to evaluate: pain intensity, pain frequency, standard of living, fear of falling, anxiety and depression.

Data acquisition of the patient involved the hardware components and also the development of a special purpose software programmed to collect data from the distinct sensors and devices for analysis.

\section{First Immersive Virtual Environment-Based Study}

a) Primary: evaluation of the effectiveness of a rehabilitation program based on virtual immersion over the patients' gait with PD on a short term.

b) Secondary: evaluation of the effectiveness of a rehabilitation program based on virtual immersion over functionality about patients with PD on a short term.

\section{Second Immersive Virtual Environment-Based Study}

a) Primary: evaluation of the effectiveness of a rehabilitation program based on virtual immersion over short term PD patients' gait.

b) Secondary: To evaluate the effectiveness of a rehabilitation program based on virtual immersion on functionality about patients with PD on a short term.

Table I describes the variables used in the above described studies.
TABLE I. Variables Studied in Both Traditional Observational Study, AND ViRTUAL IMMERSION

\begin{tabular}{|c|c|c|c|}
\hline Variables & Study 1 & Study 2 & Study 3 \\
\hline \multicolumn{4}{|l|}{ Motor variables } \\
\hline Gait & v & $\mathrm{v}$ & $\mathrm{v}$ \\
\hline Stride length & $\mathrm{v}$ & $\mathrm{v}$ & $\mathrm{v}$ \\
\hline Stride height & $\mathrm{v}$ & $\mathrm{v}$ & $\mathrm{v}$ \\
\hline Stride width & $\mathrm{v}$ & $\mathrm{v}$ & $\mathrm{v}$ \\
\hline Stride rhythm & $\mathrm{v}$ & $\mathrm{v}$ & $\mathrm{v}$ \\
\hline Stride frequency & $\mathrm{v}$ & $\mathrm{v}$ & $\mathrm{v}$ \\
\hline Arm swing & $\mathrm{v}$ & $\mathrm{v}$ & $\mathrm{v}$ \\
\hline Functionality & v & $\mathrm{v}$ & $\mathrm{v}$ \\
\hline Freezing & $\mathrm{v}$ & $v$ & $\mathrm{v}$ \\
\hline Gait Freezing & v & $\mathrm{v}$ & $\mathrm{v}$ \\
\hline Manual freezing & v & $v$ & $v$ \\
\hline $\begin{array}{l}\text { Freezing in a virtual favourable } \\
\text { environment }\end{array}$ & v & $v$ & $v$ \\
\hline $\begin{array}{l}\text { Freezing in a virtual provocative } \\
\text { environment }\end{array}$ & v & $v$ & $v$ \\
\hline Balance & v & $\mathrm{v}$ & $\mathrm{v}$ \\
\hline \multicolumn{4}{|l|}{ Non-motor variables } \\
\hline Pain intensity & $\mathrm{v}$ & $\mathrm{v}$ & $\mathrm{v}$ \\
\hline Pain Frequency & $\mathrm{v}$ & $v$ & $v$ \\
\hline Standard of living & 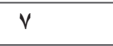 & $\mathrm{v}$ & $v$ \\
\hline Fear of falling & $\mathrm{v}$ & $v$ & $v$ \\
\hline Anxiety & v & $\mathrm{v}$ & $\mathrm{v}$ \\
\hline Depression & $\mathrm{v}$ & $v$ & $\mathrm{v}$ \\
\hline Sleep disturbance & $\mathrm{v}$ & & \\
\hline
\end{tabular}

Experimental studies with virtual immersion took about five weeks with over 15 sessions, whose protocol is as follows:

- Week 1-2: Oculus Rift plus in a sitting exercise.

- Week 3: Oculus Rift plus standing exercise.

- Week 4: Oculus Rift, standing exercise plus gait exercise.

- Week 5: Oculus Rift, standing exercise plus gait exercise with obstacles.

Without an in depth analysis on the protocol applied by the medical research personnel [21], a number of comparative analysis of the studies over two different population groups were performed, one applying the observational study and other implementing virtual immersion techniques, both in a favourable immersive environment (with both visual and acoustic stimuli, for example: an open space free of obstacles) and the other being a challenging environment (with both visual and acoustic stimuli that may cause stress in the patient).

\section{B. VR/NI}

To evaluate the freezing phenomenon through patient training using VR/NI systems, special software components, akin to these technologies have been developed. In order to achieve this goal, the features of the environment should meet at least one of the following requirements:

- Easy to control: intuitive control with simple movements or gestures. The system returns a response in accordance with the programmed activity, once it detects the movements thus creates a visual feedback for the environment management.

- Attractive/Motivating: development of a training application to be installed on a personal computer and whose handling does not require advanced computer skills.

Hence, three software components have been developed, directly related to the training and monitoring of patients: (1) editor, used by 
researchers for editor layout; (2) environment launcher, wherein the stage is configured to increase or decrease the stress provocation and (3) a monitoring module, for further analysis of the information generated during training.

The scenarios built with the environment editor are intended to evaluate the variables listed above applied to gait, manual dexterity and freezing:

- Gait evaluation: the VR, Oculus Rift goggles, NI device and Kinect were used for that purpose, to accomplish analysis of all the variables related to the gait.

Kinect includes an infrared light grid and a video camera capable of generating a 3D map of the patient's physiognomy, albeit with limited accuracy. However, the depth sensor is capable of capturing the patient's position and submitting the information to the database, delivering professionals a wide variety of information regarding postural control, thus endorsing the potential of this tool to evaluate posture in a clinical environment.

- Manual dexterity: Finger pinch exercise is used to evaluate this. The participant is to try catch with his dominant hand a certain object between his index and thumb fingers, performing variations of speed and pressure. This exercise can be parameterized with multiple levels of speed. For this, a developed component using the Leap Motion device was used, which controls the movements of the hand with sufficient precision to determine the movement variations.

- Freezing: for the evaluation of freezing, Oculus Rift, Kinect and Leap Motion were used. To do this, they have carried out the tasks called "Stepping in place task”, “Tapping task” and balance training.

- Stepping in place task: is a task developed by Nantel et al. [22] to provoke and evaluate freezing and the progress of patients with PD with a $93 \%$ specificity and $87 \%$ sensitivity respectively for freezing detection. The task involves the patient, in standing position, raises his knees alternately remaining in the same spot. The task begins with the patient standing and after ten seconds, an audio signal is used to indicate the patient to start raising knees alternately for a hundred seconds.

- Tapping Task: has been used with various motifs throughout history. In this research study this task is used to evaluate the manual freezing according to Ziv et al. [23]. The task consists on the participant having to move only the index finger flexion and extension of the metacarpophalangeal joint as quickly as possible for fifteen seconds.

- Balance: Rated by Tinetti Scale [24]. It is a hetero-administered questionnaire consisting on 9 aspects related to equilibrium and 7 related to gait. The score is rates 0 for subjects who fail to or just maintain balance, one for those performing the movement with compensation and 2 for those acquiring the correct position without difficulty. The maximum score is 16 for balance and 12 for gait, the combined of both is a total score of 28. It's considered that between 19-24 risk for fall is minimal, whereas $<19$ implies a high risk of fall.

Medical researchers were in charge of patients' personal data management as part of their studies which has been done according to compliance with the Level 3 of LOPD (Spanish Organic Law of Data Protection): patient information is for exclusive use of the research team and anyone outside the project can't access such information, except the Ministry of Economy and Competitiveness, founders of the study and Ethics Committee for Clinical Research of HULP, or any other competent authority in the matter and that requires at any time.

Patient's information concerning the interviews (patients' assigned code, gender, age, and years of evolution of PD medication since the last time) has also been stored. Several questions regarding the criteria for inclusion and exclusion and Mini-mental State Examination were also included. Scores were collected from the following evaluation scales of non-motor signs: EVA and frequency of pain. All remaining results of evaluation of non-motor signs were recorded in the questionnaire completed by the patient himself. Measurements of motor signs, except for the valuation of balance, were all digitally recorded in the database. The balance evaluation was recorded in the own Tinetti scale.

The information used for cognitive inference has been made anonymous, impeding any information disclosure through informatic data leakage or any other manner, according to LOPD.

\section{Cognitive Motor}

In a final phase of the project it was included as an additional component of special interest: a cognitive inference engine, so that data taken during the course of the exercises with patients stored could be classified, easier to use and distribute through scientific community for analysis. The goal of integrating a cognitive engine is to provide the platform for a standardised basis for measures to allow comparison of the variables taken as well as the different factors that impact exercises and allow the system to:

- Obtain correlations that could have been overlooked by the medical research personnel.

- Allow communication and information exchange between entities, allowing different hospitals and organisations the location and analysis of the data obtained.

- Extrapolate the results corresponding to different pathologies, extending the analysis and diversifying the platform and original study.

- Write reports based upon any existing measure on the system.

As a basis for the analysis of information collected in the training of patients, the results of the application of descriptive statistical methods on socio-demographic, motor and non-motor variables were used. For confirmation of normal distribution of the data the KolmogorovSmirnoff test was applied.

The analysis of variance (ANOVA) approach is used to analyse each stage group factor as PD. The normal distribution of the data was calculated using the Kolmogorov-Smirnov test: most of the variables correlated to a Gaussian distribution. The descriptive results of the study were represented by statistical frequency and percentages for qualitative variables and media with its standard deviation (SD) for the quantitative variables.

Both records of the variables studied during training and statistical information were introduced for creating rules and evaluation and perform the inference of knowledge, in order to:

- Analyse and interpret data and help medical research to decisionmaking regarding the evaluation of the evolution of $\mathrm{PD}$, and especially the progress of the phenomenon of freezing.

- Using their own experience, based on the results obtained by researchers using machine learning (ML).

- Interaction with researchers through natural language, for increasing the understanding between the researcher and the system.

\section{Analysis of Existing Solutions}

Cognitive computing allows application to process regarding pattern recognition, data mining and machine learning, applied in this project to extract new knowledge continuously from the information gathered by investigators in the phases of training patients. This was done using interaction processes in natural language to ease interaction with the researches, i.e., the so-called virtual assistants.

Over the course of the project, different proposals were analysed, 
these came from systems applying cognitive computing to create virtual assistants. Among the possibilities that can be found:

- IBM Watson suggests services focused on language processing. Watson Conversation (WCS) allows automation of the interactions between users and systems. Using WCS, users can define aspects such as NLP intentions and entities and simulate complete conversations. WCS is used with other NPL Watson services such as Watson Natural Language Classifier and Watson Discovery.

- Microsoft LUIS (Language Understanding Intelligence Service) is a component of Microsoft Cognitive (MCS) services used to create and process natural language models. LUIS provides a sophisticated toolkit that enables developers to train the new conversational platform models. LUIS can also be used with other word processing API in MCS, for example, Linguistic Analysis and Text Analytics.

- Google Natural Language API as a part of Google Cloud focuses on NLP and NLU. NL API provides the ability to detect intentions and entities, feeling analysis, classification of content and relationship graphs. The NL API also includes sophisticated tools for training and new NL authoring models.

- Facebook Wit.ai is the platform behind the NLP/NLU capabilities of the Facebook Messenger platform. One of the best qualities of Wit.ai is the toolkit that can be used to train new models and monitoring interactions between users and the platform.

- Amazon Lex/Amazon Alexa Skills Kit enables the definition of intent and relevant entities in conversational interactions. One of the great advantages of Alexa is its integration with other web services such as Amazon offers, such as AWS Lambda or AWS Cognito.

- Google DialogFlow (known as api.ai) is a platform that allows developers to design and implement conversational interfaces that can be integrated into external applications such as chatbots. DialogFlow enables speech recognition and NLU fulfilment and a robust management toolkit.

- Rasa is the only cognitive computation system based on free software and is not offered as a cloud service. Rasa provides natural language recognition (rasa NLU) and a way to carry out complicated dialogues with ML (rasa Core).

\section{Conversational Interface Queries}

Conversational interfaces or chatbots are gaining prominence day by day thanks to the ease with which they fit into online solutions, and in some cases, without software programming skills. For this, natural language processing utilities (NLU) are mostly used.

When talking about chatbots it is important to account for the following concepts:

- NLP (natural language processing). general concept that refers to the ability of computer systems to understand the meaning of a text written in natural language.

- NLU (natural language understanding). It refers to the processes capable of decomposing unstructured text data structures that can be classified.

- NLG (natural language generation). It refers to the process followed by the chatbot to provide its response based on what has been asked.

In Table II, a comparison of the services listed in the above [25] (Watson, LUIS, API.ai, Wit.ai, Lex and RASA) for NLU module functionality that they implement, to visualise what Rasa can accomplish, offering the same functionality as the other solutions except that is not offered as a cloud service, but a downloadable application of local installation, which in turn is an advantage if what is intended is to use it within private networks.
TABLE II. Comparative OF Functionality BaSED ON THE NLU SERvices Offered by Cognitive Computing Main Solutions

\begin{tabular}{|l|c|c|c|}
\hline Service & Intents & Entities & Batch import \\
\hline LUIS & + & + & + \\
\hline Watson & + & + & + \\
\hline API.ai & + & + & + \\
\hline wit.ai & + & + & O \\
\hline Lex & + & O & - \\
\hline RASA & + & + & + \\
\hline
\end{tabular}

For Mobeeze, Rasa was chosen for the platform configuration, since its adaptability to the needs of the current project was more important than the capabilities of the other solutions.

Rasa provides software for creating virtual assistants or chatbots that allow users to perform queries through natural language. Is consists of a set of open source ML tools. The main ones are NLU Rasa and Rasa Core:

- NLU Rasa: NLP tool for intention classification and entity extractions. On the one hand, Rasa NLU applies a classification of intent, i.e., interprets the meaning of the question based on a previously conducted training that determines through a percentage the probability in intention by which the question is asked. On the other hand, data analysis is carried out using the entity extraction modulus.

- Rasa Core (or Rasa DM - dialogue management) is the module responsible of guiding the conversations, using the history and the external context of the dialogue. This solution is trained using supervised ML and interactive way in order to create sophisticated dialogs.

- NLG Rasa: This is the process that builds Rasa response to a query.

Rasa uses ML techniques for the training of its decision engine. Thus, instead of thousands of rules, Rasa learns real conversational patterns. It must also be remarked that Rasa is distributed through Apache 2.0 open source license.

Fig. 1 shows the process followed by Rasa for analysing a sample formulated query, search information and make corresponding response [26].

As an information extracting tool, spaCy [27] was used [28]. It's a ML-based algorithm library for extracting information and a training platform.

SpaCy is a Python developed library, also offering pre-trained models in different languages, dependency analysis, segmentation phrases syntax, tokenisation, etc.

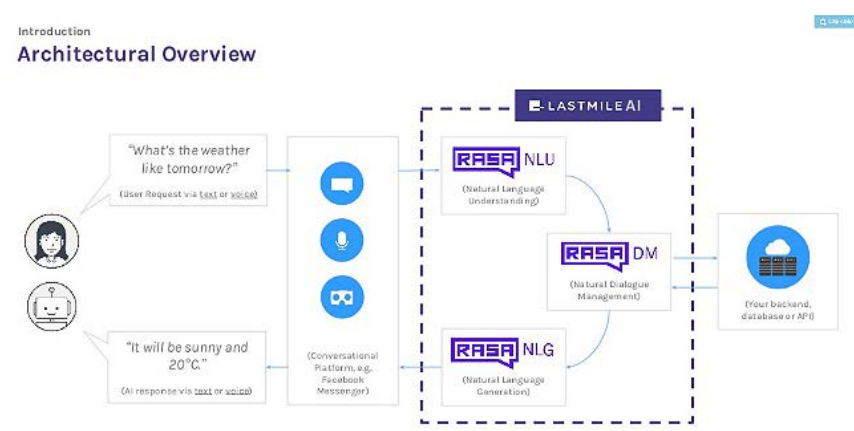

E. LASTMILE

Fig. 1. Details Component involved in Rasa. 
Regarding the data used for training, it must be kept in mind that in order check for correct functioning, both system capability data and evaluation data are required. Therefore, the existence of a considerable set of data is necessary.

For the current test, the training data set used was a corpus based on Wikipedia articles. This dataset is named Question-Answer Dataset [29]. Use of this corpus allowed having a set of data that served as a base to maintain a dialogue between the researcher and the chatbot.

Subsequently, a dataset was incorporated, this one consisting in specific information of the tests performed on the patients during Mobeeze virtual immersion training and also data from the results report submitted by the researchers.

\section{RESUlts AND Discussion}

\section{A. Immersion Virtual Patients with Parkinson}

Throughout the Mobeeze project, samples of patients diagnosed with PD have been collected. Those participants in the control group (inhibitor freezing environment) that had a homogeneous gait with respect to walking variables (angles of both hips, knees and shoulders, stride height and speed). However, those subjects who were randomized to intervention group (favourable environment freezing) made a nonconstant motion regarding the stride height and speed.

In this sense, those participants registered an increase of nearly twice the stride speed right at the moment that they came across other people or cars in the virtual environment. Furthermore, stride height is also clearly decreased prior approach to the above-mentioned virtual entities, which could be classified as a gait freezing.

In contrast, in the two immediate steps after the crossing, stride height increases significantly even in relation to the average during real world gait.

These results justify the use of the combination VR/NI to work through the freezing, coping with people or objects crossing the street in a non-invasive environment.

\section{B. Motor Cognitive Inference}

The objective pursued by the inclusion of a cognitive computing engine in the Mobeeze project is to analyse the possibility that the AI relates elements of the virtual immersion training designed as therapy for gait disturbance in PD patients.

To this end, it has been used as the basis for training the results obtained in the training stage of patients with PD in virtual immersion, added to the study conducted by the medical staff on the state of the art of the effectiveness of virtual immersion this same disease [30].

To do this, the classifications made and the results obtained for VR training groups and control groups have been used, with traditional training, based searches on scientific papers on the use of virtual immersion as therapeutic training. The methodology used followed the process indicated in Fig. 2.

The collection of information gathered by the medical team, is currently working on creating a knowledge base that provides findings (not currently validated from the medical point of view) on the effectiveness of the results of treatment in patient's PD in virtual immersion.

In Fig. 3. The results of the first tests performed using the information collected so far seen.

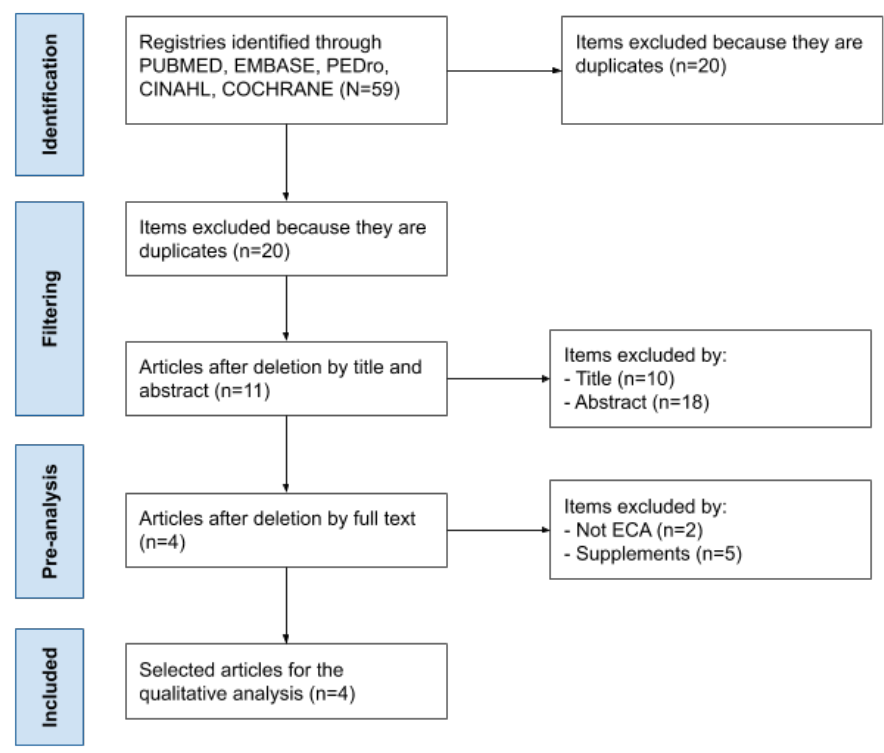

Fig. 2. Method of articles selection.

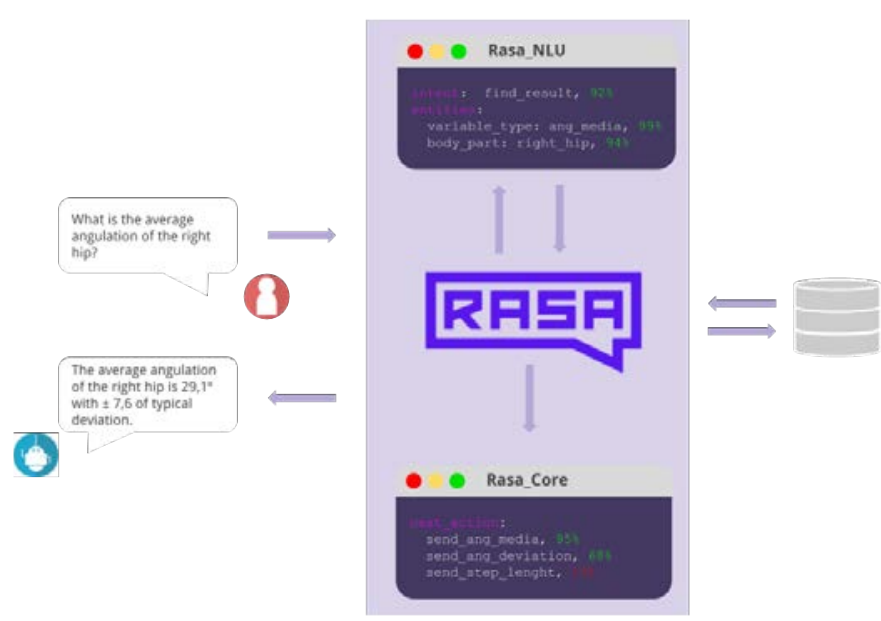

Fig. 3. Conversation example between an investigator and Rasa about the results through the training with patients.

As of today, the knowledge base is very limited, however it is expected that the incorporation of new data from training with patients and the use by researchers will help refine the answers.

\section{Conclusions and Future Lines}

A multidisciplinary project, called Mobeeze, has been developed, specially designed for people with Parkinson's disease, and that affects them with problems regarding mobility, specifically the phenomenon of freezing. In order to solve this, technologies like Oculus Rift, Microsoft Kinect or Leap Motion for recreation immersive virtual scenarios have been used. The purpose of this article is to transmit the evaluation made by the research team doctor who participated in the project on the applicability of ICT in the therapeutic treatment and tracking freezing. The results report developed by the medical team research the following conclusions are thrown:

1. The study conducted intends to describe the changes that occur in gait parameters for PD patients to undergo in two VR environments: inhibitor and freezing provocative.

2. VR therapy appears to modify the gait variables regardless of the environment in which the subject with PD is immersed. 
3. VR therapy could be an alternative treatment to improve gait in patients with PD.

Furthermore, there has been an experimental development for extracting knowledge training conducted with patients using an AI system based on RASA, an ML system which provides an information search based conversational interface.

In order to achieve efficient machine learning, information quality and efficient focus on the problem is essential. It is the case of an employee of IBM, Jonathan Rezek, who works in the computer business of IBM promoting Watson [31] [32], who it turned out suffered from $\mathrm{PD}$. To reduce the symptoms of PD, the main symptomatic therapy is L-DOPA, however, it can eventually cause dyskinesia [33]. Knowing the possibilities offered by cognitive engines, in this case Watson, contacted the medical team who treated him to carry out drug discovery that could be applied to treat dyskinesia, although originally were indicated for other diseases. The medical team gathered information training Watson and the results were encouraging as five drugs that preliminary tests showed positive results against dyskinesia, which had not been previously considered in patients with PD were found.

The first results obtained using Mobeeze in those affected with this disorder; suggest that virtual immersion when making therapy for this disorder may be beneficial. However, the project is still at a very early stage of training and requires additional tests on patients with PD, therefore it is too early to confirm its effectiveness.

\section{A. Limitations}

Introduction of the cognitive inference engine as an additional component to the project Mobeeze has led to the lack of initial data. This has resulted in a very early prototype calibration, which rendered the project results inconclusive.

\section{B. Future Lines}

As a continuation of the project, the intention is to continue the same line in a way that could enable the acquisition of new knowledge, on one hand, through training realized by the medical team that could bring a volume of information for patients with more population, group, major diversification, or the inclusion of other variables that enquire the base of knowledge of the interference motor, like the embedment of a new knowledge for the corpus data obtained by the state of art based on publications realized by other investigation teams.

The future lines in this work are directed to the study of the use of this platform through new technologies based on the speech recognition (like Alexa, Google Assistant etc.) and how to adapt it in order to carry out the therapeutic training through the virtual immersion for this disorder, in a way that could communicate with the patient during this treatment. This new panorama presented with this technology makes it easy to treat the patient by adding the virtual environment new acoustic stimuli and new study variables for a defined therapy that will be closer to reality.

\section{ACKNOWLEDGEMENT}

This project has been funded by the Ministry of Economy and Competitiveness. It has been a collaborative project between Gestor. ex, Virtualware, Fidesol and IDIPAZ. Project code: RTC-2015-3870-1.

\section{REFERENCES}

[1] Emamzadeh FN, Surguchov A. Parkinson's Disease: Biomarkers, Treatment, and Risk Factors. Front Neurosci. 2018; 12:612. 2018.

[2] Holden MK. Virtual environments for motor rehabilitation: review. CyberpsycholBehav 2005; 8: 187-211.

[3] Rizzo A, Kim GJ. A SWOT analysis of the field of virtual reality rehabilitation and therapy. Presence 2005; 14: 119-46.

[4] Viau A, Feldman AG, McFadyen BJ, Levin MF. Reaching in reality and virtual reality: a comparison of movement kinematics in healthy subjects and in adults with hemiparesis. J NeuroengRehabil 2004; 1: 11.

[5] Subramanian S, Knaut LA, Beaudoin C, McFayden BJ, Feldman AG, Levin MF. Virtual reality environments for post-stroke arm rehabilitation. J NeuroengRehabil 2007; 4: 20.

[6] Kuttuva M, Boian R, Merians A, Burdea G, Bouzit M, Lewis J, et al. The Rutgers Arm, a rehabilitation system in virtual reality: a pilot study. CyberpsycholBehav 2006; 9: 148-51.

[7] Fulk GD. Locomotor training and virtual reality-based balance training for an individual with multiple sclerosis: a case report. J Neurol Phys Ther 2005; 29: 34-42.

[8] Holden M, Dettwiler A, Dyar T, Niemann G, Bizzi E. Retraining movement in patients with acquired brain injury using a virtual environment. In Westwood JD, Hoffman HM, Mogel GT, Stredney D, eds. Medicine meets virtual reality. Newport Beach: IOS Press; 2009. p. 192-8.

[9] You SH, Jang SH, Kim Y, Hallett M, Ahn SH, Kwon Y, et al. Virtual reality-induced cortical reorganization and associated locomotor recovery in chronic stroke. An experimental- blind randomized study. Stroke 2005; 36: 1166-71.

[10] Fidopiastis CM, Stapleton CB, Whiteside JD, Hughes CE, Fiore SM, Martin GA, et al. Human experience modeler: context driven cognitive retraining to facilitate transfer of learning. CyberpsycholBehav 2006; 9: 183-7.

[11] Van Gemert-Pinjen L, Kelders S, Kip H, Sandderman R. eHealth research, theory and development: a multi-disciplinary approach. Routledge; 2018.

[12] Moya, P. Kinect para ayudar a pacientes de Parkinson con bloqueo de la marcha. Omicromo. https://omicrono.elespanol.com/2015/05/kinect-parapacientes-de-parkinson/ (10 May 2015)

[13] Pilgrim, Tim. Novel Kinect system helps keep Parkinson's patients moving. Phys.org. https://phys.org/news/2018-05-kinect-parkinsonpatients.html (24 May 2018)

[14] Alvarez M, Grogan P. Connecting with Kinect ${ }^{\circledR}$ To Improve Motor and Gait Function in Parkinson Disease. Neurology Center for Neurological Care and Research San Antonio TX. April 2012.

[15] Dockx K, Bekkers EMJ, Van den Bergh V, Ginis P, Rochester L, Hausdorff JM, Mirelman A, Nieuwboer A. Virtual reality for rehabilitation in Parkinson's disease. Cochrane Database of Systematic Reviews 2016, Issue 12. Art. No.: CD010760.

[16] Evans, D. (2017). The fundamental differences between automation and AI. VentureBeat, 4 October. https://venturebeat.com/2017/10/04/ the-fundamental-differences-between-automa-tion-and-ai/. Accessed 5 January 2018.

[17] IBM Watson: la tecnología cognitiva que abre una nueva era de la computación. Ver en: https://www-03.ibm.com/press/es/es/ attachment/49310.wss?fileId=ATTACH_FILE1\&fileName=Dossier\%20 IBM\%20Watson.pdf

[18] Krishna, Chico. Harnessing AI for Parkinson's Research. https://www. michaeljfox.org/foundation/news-detail.php?harnessing-ai-for-parkinsonresearch (14 January 2019).

[19] IBM presenta pequeño sensor de uñas con inteligencia artificial y anuncia una alianza con la Fundación Michael J. Fox para comprender mejor y predecir la progresión de la enfermedad de Parkinson (8 January 2019). Retrieved from: https://www-03.ibm.com/press/mx/es/ pressrelease/54644.wss

[20] Lombardo, J. M., M. A. Lopez, V. M. García, M. López, R. Cañadas, S. Velasco, and M. León. 2018. PRACTICA. A Virtual Reality Platform for Specialized Training Oriented to Improve the Productivity. International Journal of Interactive Multimedia and Artificial Intelligence (IJIMAI). Special Issue on Artificial Intelligence Applications. Volume 5, num. 4, pags. 94-101. ISSN: 1989-1660

[21] Morales-Gómez S, Elizagaray García I, Yepes-Rojas O, De la PuenteRanea L, Gil-Martínez A. Efectividad de los programas de inmersión virtual en los pacientes con enfermedad de Parkinson. Revisiónsistemática. Rev Neurol 2018; 66: 69-80.

[22] Nantel J, de Solages C, Bronte-Stewart H. Repetitive stepping in place identifies and measures freezing episodes in subjects with Parkinson's disease. Gait Posture 2011; 34: 329-333.

[23] Ziv I, Avraham M, Dabby R, Zoldan J, Djaldetti R, Melamed E. Early- 
occurrence of manual motor blocks in Parkinson's disease: a quantitative evaluation. Acta Neurol Scand. 1999 feb;99(2):106-11.

[24] Rodríguez Guevara C, Lugo LH. Validez y confiabilidad de la Escala de Tinetti para población colombiana. Rev ColombReumatol. 19(4):218-33.

[25] Braun, D., Hernandez Mendez, A., Matthes, F., Langen, M. Evaluating Natural Language Understanding Services for Conversational Question Answering Systems. 2017. Proceedings of the 18th Annual SIGdial Meeting on Discourse and Dialogue. Association for Computational Linguistics. Pages 174--185. DOI: 10.18653/v1/W17-5522

[26] Rasa AI: Building clever chatbots. Slideshare. Available at: https://www. slideshare.net/TomBocklisch/rasa-ai-building-clever-chatbots

[27] Davis E. King. Dlib-ml: A Machine Learning Toolkit. Journal of Machine Learning Research 10, pp. 1755-1758, 2009.

[28] Paramveer Dhillon, Dean Foster and Lyle Ungar, Eigenwords: Spectral Word Embeddings, Journal of Machine Learning Research (JMLR), 16, 2015.

[29] Noah A. Smith, Michael Heilman, and Rebecca Hwa. Question Generation as a Competitive Undergraduate Course Project in Proceedings of the NSF Workshop on the Question Generation Shared Task and Evaluation Challenge, Arlington, VA, September 2008. Available at: http://www. cs.cmu.edu/ nasmith/papers/smith+heilman+hwa.nsf08.pdf

[30] Morales-Gómez S, ElizagarayGarcía I, Yepes-Rojas O, De la PuenteRanea L, Gil-Martínez A. Efectividad de los programas de inmersión virtual en los pacientes con enfermedad de Parkinson. Rev Neurol 2018; 66: 69-80.

[31] Rezek, J. May 2018. Revolutionizing drug research for Parkinson's with AI. AI/Watson | Healthcare. IBM. Available at: https://www.ibm.com/ blogs/client-voices/revolutionizing-drug-research-parkinsons-ai/

[32] Rezek, J. How one Parkinson's patient drove a groundbreaking research effort. Passion Projects. IBM. Available at: https://www.ibm.com/thoughtleadership/passion-projects/parkinsons-watson-drug-discovery/

[33] Lee CS. Levodopa-induced dyskinesia: Mechanisms and management. Issue: BCMJ, Vol. 43, No. 4, May 2001, page(s) 206-209 Articles. Available at: https://www.bcmj.org/articles/levodopa-induced-dyskinesiamechanisms-and-management

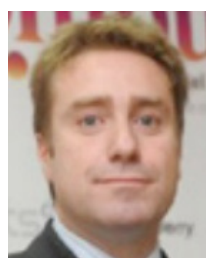

Juan Manuel Lombardo

Juan Manuel Lombardo, PhD in Computer Science from the Pontifical University of Salamanca, was graduated in Economics and Business Administration in the University of Granada, Spain, Diploma of Advanced Studies (DEA) in Economics from UNED, Research Sufficiency in Business Science from the Complutense University of Madrid and Diploma of Advanced Studies (DEA) in Sociology from the Pontifical University of Salamanca. He is CEO at Fidesol and Professor at Andalusia Business School. Dr. Lombardo is the author of numerous articles and research papers published in journals and books of national and international conferences. Visiting Professor at the Private Technical University of Loja (UTPL Ecuador), The National University of the Northeast (Argentina), University Francisco José de Caldas (Colombia), Catholic University of Colombia, Catholic University of Ibarra (Ecuador), University of Lisbon (Portugal) and National Engineering University (Peru). Member of the Knowledge Management committee of AEC (Spanish Association for Quality) and the Institute CICTES (Ibero-American Centre on Science, Technology and Society).

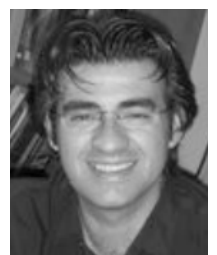

Miguel Ángel López

Miguel Ángel López, has a degree in Engineering in Technical Engineering in Computer Systems from the University of Almería, graduates in Computer Engineering and Master in Softcomputing and Intelligent Systems from the University of Granada. Currently he is CTO. at Fidesol where performs different roles on the projects. His research focuses on distributed systems, management, integration and analysis of data, robotics, fuzzy logic systems, and the development of virtual reality environments for different purposes.

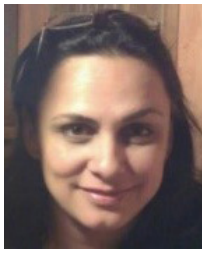

\section{Mabel López}

Mabel López has a degree of Computer Science Engineering. She is Knowledge Manager at Fidesol. Participates in the research and development strategy of this entity, technology transfer and analysis of technological trends, such as big data, internet of things, virtual reality, cognitive engines, machine learning, etc. Currently, she is involved in several $\mathrm{R}$ \& D projects related to the mentioned technologies.

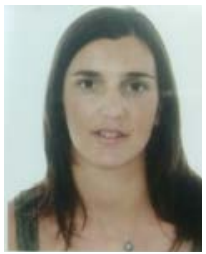

Mónica León

Mónica León, Degree in Documentation from the University of Extremadura and Diploma in Library Science from the University of Granada. He is currently a technician in the R \& D Transfer Office of the Free Software R \& D Foundation, performing tasks of technology surveillance, coordination and management of proposals for public incentives for $\mathrm{R}$ \& D projects, throughout the chain of development of the projects -from the call to the final justification-, and the search, constitution and coordination of partners and consortiums for collaborative projects.

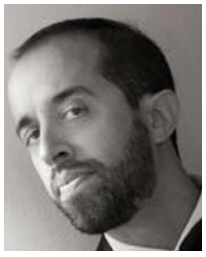

Felipe Miron

Felipe Miron is the European Projects Coordinator of the KTO at FIDESOL, where he manages the foresight and technological surveillance actions of the entity, both state and European level, as well as the project proposals. He is a Computer Engineer from the University of Granada. Before joining FIDESOL, he worked for the Government of the Region of Murcia leading a development team for the transformation of the File Processing system of the Labor Counseling and Social Policy. He has been part of development teams for national projects (Banesto, Telefonica, Onda Cero) and international (Credit Suisse, IBM). He has collaborated in several publications related to disciplines such as Artificial Intelligence, Mobility and Big Data.

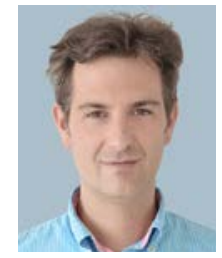

Jon Arambarri Basanez

Jon Arambarri Basanez. Dr. Telecommunication Engineer and MBA. Responsible for Innovation at www. virtualwaregroup.com oriented to the generation of new product for eHealth and Industry4.0. My research interests combine the regulation and efficient management of Telecommunications Infrastructures (Telecommunication Networks, Visualization and Multimedia and Internet of Things - IoT) together with the optimization of ICT services (Artificial Intelligence and Predictive Systems, Cyber Security) the development of innovative business solutions, mainly for eHealth and industry4.0.

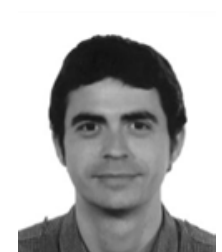

David Alvarez

David Alvarez receibed his Ph.D in Computer Science at the University of León, Spain in 2015, during which he worked on an Optical Caracter Recognition of handwritten documents. He then joined the Research Group on Numerical Simulation and Scientific Calculus (SINUMCC) where he worked on Forest Fire Spread Simulation. He is now a member of the Research Group of Fundación del Software Libre (FIDESOL). His research interes include in general computer vision, machine learning, image recognition and pattern recognition. 